\title{
Nanoscale Soldering of Positioned Carbon Nanotubes using Highly Conductive Electron Beam Induced Gold Deposition
}

Madsen, Dorte Nørgaard; Mølhave, Kristian; Mateiu, Ramona Valentina; Bøggild, Peter; Rasmussen, A.M.; Appel, C.C.; Brorson, M; Jacobsen, C.J.H.

\section{Published in:}

Proceedings of the Third Conference on Nanotechnology

Link to article, DOI:

10.1109/NANO.2003.1231786

Publication date:

2003

Document Version

Publisher's PDF, also known as Version of record

Link back to DTU Orbit

Citation (APA):

Madsen, D. N., Mølhave, K., Mateiu, R. V., Bøggild, P., Rasmussen, A. M., Appel, C. C., Brorson, M., \& Jacobsen, C. J. H. (2003). Nanoscale Soldering of Positioned Carbon Nanotubes using Highly Conductive Electron Beam Induced Gold Deposition. In Proceedings of the Third Conference on Nanotechnology (Vol. 2). IEEE. https://doi.org/10.1109/NANO.2003.1231786

\section{General rights}

Copyright and moral rights for the publications made accessible in the public portal are retained by the authors and/or other copyright owners and it is a condition of accessing publications that users recognise and abide by the legal requirements associated with these rights.

- Users may download and print one copy of any publication from the public portal for the purpose of private study or research.

- You may not further distribute the material or use it for any profit-making activity or commercial gain

- You may freely distribute the URL identifying the publication in the public portal 


\section{Nanoscale Soldering of Positioned Carbon Nanotubes Using Highly Conductive Electron Beam Induced Gold Deposition}

\author{
Dorte Nørgaard Madsen, Kristian Mølhave, \\ Ramona Mateiu, Peter Bøggild \\ Mikroelektronik Centret \\ Technical University of Denmark \\ DK-2800 Kgs. Lyngby, Denmark \\ dnm@mic.dtu.dk
}

\author{
Anne Marie Rasmussen, Charlotte Clausen Appel, \\ Michael Brorson, Claus J. H. Jacobsen \\ Haldor Topsøe A/S \\ Nymøllevej 55 \\ DK-2800 Lyngby, Denmark
}

\begin{abstract}
We have developed an in-situ method for controlled positioning of carbon nanotubes followed by highly conductive contacting of the nanotubes, using electron beam assisted deposition of gold. The positioning and soldering process takes place inside an Environmental Scanning Electron Microscope (ESEM) in the presence of a source of gold-organic precursor gas. Bridges deposited between suspended microelectrodes show resistivities down to $10^{-4} \Omega \mathrm{cm}$ and Transmission Electron Microscopy (TEM) of the deposits reveals a dense core of gold particles surrounded by a crust of small gold nanoparticles embedded in a carbon matrix. Nanoscale soldering of multiwalled carbon nanotubes (MWNT) onto microelectrodes was achieved by deposition of a conducting gold line across a contact point between nanotube and electrode. The solderings were found to be mechanically stronger than the carbon nanotubes. We have positioned MWNTs to bridge the gap between two electrodes, and formed soldering bonds between the tube and each of the electrodes. All nanotube bridges showed ohmic resistances in the range $10-30 \mathrm{k} \Omega$. We observed no increase in resistance after exposing the MWNT bridge to air for days.
\end{abstract}

Keywords - electron beam deposition, carbon nanotubes, nanoelectronics

\section{INTRODUCTION}

Due to their unique mechanical and electrical properties, carbon nanotubes have attracted considerable attention as nanoscale building blocks for e.g. carbon-based nanoelectronics [1]. In order to investigate the potential of carbon nanotubes, various methods for forming electrical and mechanical contacts to nanostructures have been employed, and functional carbon nanoelectronic circuit components have been demonstrated [2-7]. Integrating nanoelectronic components in micro- or nanoelectronic circuitry demands a method for positioning the nanostructure and forming a reliable mechanical and electrical contact to it. In this paper we present an in-situ method for attachment and electrical contacting of nanoscale components. We demonstrate controlled positioning of carbon nanotubes on microfabricated electrodes, followed by mechanically strong and highly conductive contacting of the nanotubes as illustrated in fig. 1 . The technique does not require electrical contact to electrodes or component and allows for assembly of 3D structures.

\section{EXPERIMENTAL SETUP}

We performed the positioning and soldering process in a Philips XL30 ESEM-FEG environmental scanning electron microscope with water vapor as imaging gas. Typically the microscope was operated at a water vapor pressure of $100 \mathrm{~Pa}$ and a beam acceleration voltage of $10 \mathrm{kV}$. When introducing a metal-organic precursor gas into the E-SEM chamber, the electron beam locally decomposes the metal-organic compound and thereby deposits a metal-containing material on a sample surface at the focus point of the e-beam [8]. As more material is added, the deposit grows as a column of material in the direction of the beam. We used the compound Dimethylacetylacetonategold(III), which has a vapor pressure of $1 \mathrm{~Pa}$ at $25^{\circ} \mathrm{C}$. The gold compound was placed close to the sample, in a container with a narrow bore tube for controlling the flow of vapor out of the source. Using a $2 \mathrm{~mm}$ long tube with a diameter of $0.8 \mathrm{~mm}$, we obtained a growth rate of $500 \mathrm{~nm} / \mathrm{min}$. Vertical tips taller than $15 \mu \mathrm{m}$ could be grown without significant decrease of the growth rate. In a high vacuum SEM the sample is often contaminated by carbon deposits during imaging. Using a water atmosphere in the ESEM, we did not observe any deposition when the source of the gold compound was removed from the E-SEM chamber.

Using chemical vapor deposition (CVD) we grew samples of free-standing multiwalled carbon nanotubes on a silicon substrate, following a procedure similar to the method

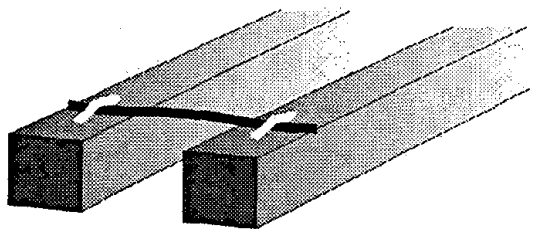

Figure 1. Sketch of a carbon nanotube positioned across two microelectrodes, soldered on to the electrodes and cut at their outer edges. 


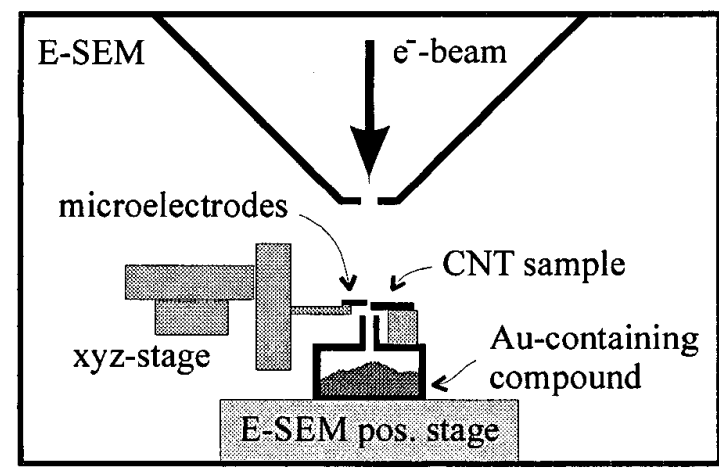

Figure 2. Setup for positioning and soldering of carbon nanotubes onto microelectrodes inside an E-SEM. Precursor gas source (Dimethylacetylacetonategold(III)) and carbon nanotube sample are placed on the positioning stage of the E-SEM, while the microelectrodes are mounted on a 3-axis nanometer precision stage.

described in the work by Xianbao et al. [9]. SEM and TEM analysis showed well graphitised MWNTs with diameters in the range $50-120 \mathrm{~nm}$ and lengths up to $40 \mu \mathrm{m}$. Some of the tubes extended over the edge of the silicon support and were thereby easily accessible.

The nanotubes were to be soldered onto microelectrodes. With standard microfabrication techniques we produced goldcoated silicon chips with multiple microcantilever electrodes extending over the edge of the chip [10]. The microelectrode chip was mounted on a nanometer precision three-axis translation stage inside the SEM while the MWNT sample was placed close to the opening of the gold-compound source, which was mounted on the position stage of the E-SEM. Thereby we could move the nanotube sample and the electrode chip in three dimensions relative to each other and to the ebeam. The setup is illustrated in fig. 2 .

\section{RESULTS}

During the positioning and soldering procedure the microelectrodes were connected to a DC voltage source and the current running between the electrodes was monitored continuously. The electrode pair was positioned so that a nanotube extending from the sample was lying across the electrodes, bridging the gap between them. By slowly scanning the beam across the nanotube at the point of contact between the nanotube and one of the electrodes, a gold-containing soldering bond was formed. To avoid unwanted contamination of the nanotubes by soldering material, we avoided imaging the suspended part of the nanotubes at high magnification. Electrical contact was established during the soldering process at the second electrode. Fig. 3 shows the current measured during the second soldering with a bias voltage of $10 \mathrm{mV}$. In this example the current rises fast to $800 \mathrm{nA}$ and then to $1100 \mathrm{nA}$ where it stabilizes. In another experiment it rose in two steps, first to about $60 \mathrm{nA}$ and then abruptly to $300 \mathrm{nA}$. A linear current-voltage curve was measured in all studied samples, indicating ohmic conduction. An additional deposition, performed with a higher beam current at the outer edge of the microelectrodes, allowed us to mechanically break

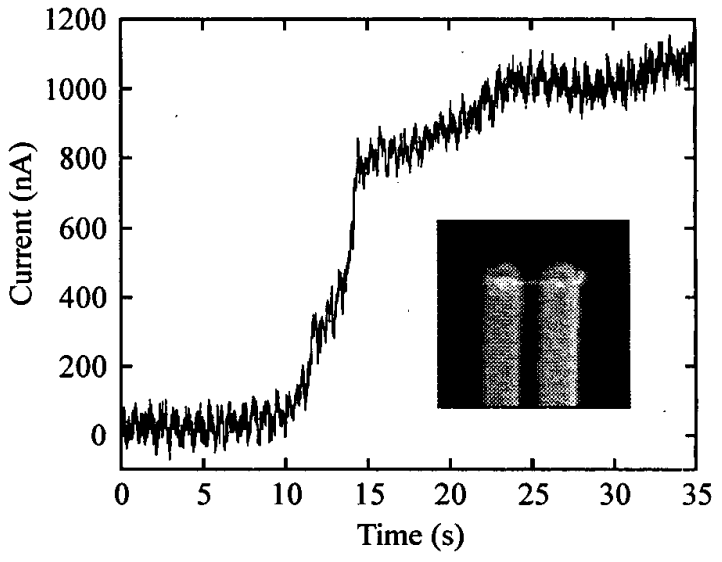

Figure 3. Current versus time during soldering of the nanotube onto the second microelectrode. A voltage of $10 \mathrm{mV}$ was applied across the electrodes. The current increased in several steps until it stabilized at $1100 \mathrm{nA}$. The device is shown in the inset.

off the MWNT parts extending beyond the electrodes, without damaging the soldering bonds. A sequence of images illustrating the positioning and soldering process is presented in fig. 4

We constructed four nanotube-bridge devises and obtained every time reliable ohmic contacts upon soldering to the second electrode. The devices showed resistances of 9, 11, 27 and $29 \mathrm{k} \Omega$, respectively, with no clear correlation to the length of the MWNT bridge. The resistances of the nanotube bridges were unaffected by breaking of the nanotube extensions as well as by deposition of the additional solderings, and were found to

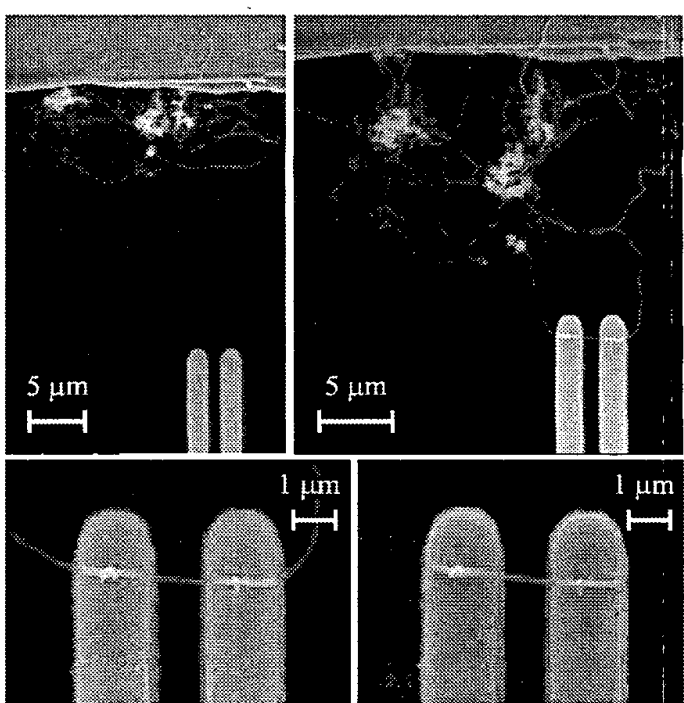

Figure 4. Positioning, soldering and release of a carbon nanotube bridge device. A microelectrode pair is positioned so that a nanotube bridges the gap and the nanotube is soldered onto both of the electrodes (Upper panel). With an additional deposition, performed with a higher beam current at the outer edge of the electrodes, the device could be released. The lower shows the device before (left) and after (right) release from the nanotube extensions. 


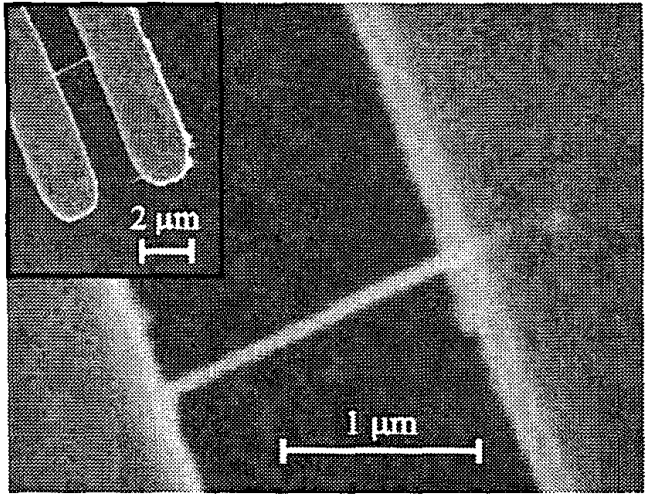

Figure 5. SEM images of soldering material deposited between microelectrode pairs. All deposited bridges with similar dimensions showed resistances of about $2 \mathrm{k} \Omega$. The bridges verifies that the soldering material is conducting.

be constant over days in air.

In order to verify that the soldering material is conducting, we deposited bridges of the material between microelectrode pairs and measured the IV characteristics. The inset of fig. 5 presents a SEM overview image of a bridge connecting the electrodes, while the main figure shows a close-up of such a bridge. We fabricated several bridges with dimensions similar to the one shown. They all showed ohmic resistances of about $2 \mathrm{k} \Omega$, which decreased over time when passing current through the bridge. Estimating the cross section from the SEM images we obtain resistivities down to $10^{-4} \Omega \mathrm{cm}$, consistent with measurements on bridges with different dimensions [11]. We also attached MWNTs onto microelectrodes by deposition of carbonaceous material originating from the background gas in a high vacuum SEM. These devices showed electrical conduction in the $\mathrm{M} \Omega$ range, thereby indicating that the metal content of the soldering material is necessary for good electrical contact.

We studied the electron beam deposited gold material further by depositing material in vertical tips suitable for TEM. The analysis revealed a crust of small $3-10 \mathrm{~nm}$ gold particles embedded in a carbon matrix, surrounding a solid core of gold with single crystalline areas of about $30 \mathrm{~nm}$. Fig. 6 presents a TEM image of such a deposited tip, showing the dense gold core and the crust with the gold nanoparticles. The inset displays a close-up of the border between core and crust, where the lattices of nanoparticles and gold core are resolved. The composition of the deposit, the size of the gold core, nanoparticles and crust thickness, depends on the deposition conditions [12].

In addition, we investigated the soldering process by depositing beams of soldering material on suspended carbon nanotubes and studying the soldering region on the nanotubes using TEM. The TEM image displayed in fig. 7 shows two such soldering regions. Gold soldering material is seen to gather around the nanotube in the soldering region and the nanotube appears locally to have a reduced diameter or to be collapsed. However, when performing the soldering on a microelectrode gold surface the deposition conditions are

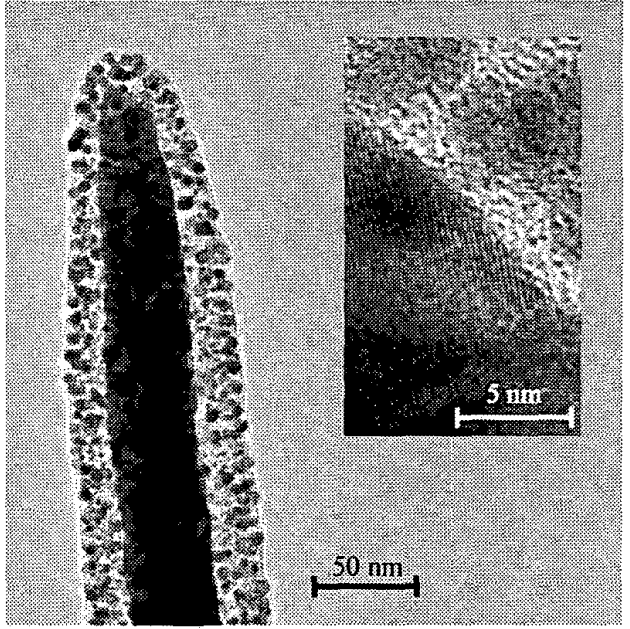

Figure 6. TEM image of a vertically deposited tip of soldering material. The tip consists of a solid polycrystalline gold core surrounded by a crust of nanosized gold particles embedded in a carbon matrix. In a close-up of the border between core and crust (inset) the lattices of the gold particles and the gold core are resolved.

different and may lead to another structure. A diameter reduction has been observed in carbon nanotubes exposed to electron irradiation and has also been predicted to occur under ion irradiation [13,14]. However, the damage threshold for electron irradiation was found to be in the order of $100 \mathrm{keV}$. Nanotube and deposits are both covered by a carbonaceous contamination layer. By replacing the open precursor gas source with a controlled inlet of precursor gas, that can be switched off, we expect to be able to strongly reduce the contamination.

Finally, we tested the mechanical strength of the solderings. In the experiment illustrated in figure 8 , we soldered a multiwalled nanotube extending from a substrate onto a microelectrode. When moving the electrode away from the substrate, thereby stretching the nanotube, we observed that the nanotube and not the soldering bond broke (a). After soldering

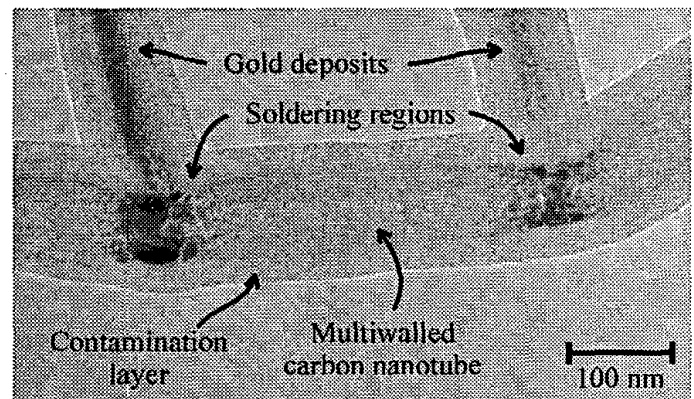

Figure 7. TEM image of deposition of soldering material onto suspended carbon nanotubes. Gold soldering material is seen to gather around the nanotube in the soldering regions and the nanotube is locally deformed. A carbonaceous contamination layer is covering tube and deposits. By a controlled inlet of precursor gas we expect to be able to strongly reduce the contamination. 
the ends of the nanotube together (b), we saw again that withdrawal of the electrode broke the tube and not any of the soldering bonds (c). Repeating the experiment, we consistently observed the nanotube to break rather than the soldering bonds, both for tube-electrode and for tube-tube bonds.

\section{Discussion}

Electron transport in multiwalled carbon nanotubes is not fully resolved and the question regarding the contribution of individual shells to the overall conductivity is still open. Frank et al. [15] observed consistent resistances of $13 \mathrm{k} \Omega$ for nearly defect-free arc-discharge grown MWNTs, whereas Collins and co-workers [16] found lower values ( $5-15 \mathrm{k} \Omega$ ) and evidence of several contributing shells at low bias voltage. We found for our four MWNT bridges resistances between 9 and $29 \mathrm{k} \Omega$. Nanotubes grown by chemical vapor deposition generally contain more structural defects than arc-discharge or laserablation grown nanotubes. These defects may cause a curving of the nanotubes (see e.g. fig. 4, upper panel). Defects are expected to decrease the conductivity of each shell. Still, the nanotube resistances obtained in our nanosoldered CVD-grown MWNTs are relatively small compared to ref. 15 and 16 . We observed that the soldering process brings gold particles in close contact with the carbon nanotube and deforms the tube in the soldering region. This suggests that the nanosoldering establishes contact to a larger number of shells, compensating for the reduced conductivity of the individual shells and thereby giving a rather low overall resistance. However, when performing the soldering on a microelectrode gold surface the deposition conditions are changed compared to soldering on suspended nanotubes and this may lead to a soldering region structure different from the structure presented in fig. 7 .

The resistivity of the soldering material is slightly larger than the value $1.3 \cdot 10^{-5} \Omega \mathrm{cm}$ reported by Bietsch et al. for pure microcontact printed gold nanowires of similar dimensions [17], and about 2 orders of magnitude larger than that of bulk gold. For electron beam deposited nanowires, resistances as small as ours have been obtained by heating the sample to $80^{\circ} \mathrm{C}$ during deposition, which increased the relative content of gold [8]. These values were obtained only after annealing at

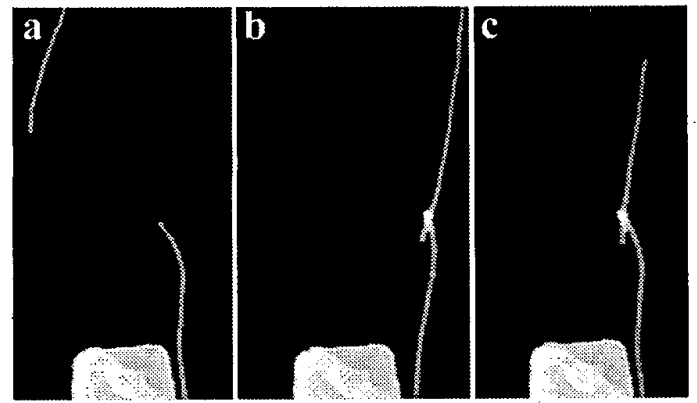

Figure 8. SEM image sequence showing tube to tube soldering. First the MWNT was soldered onto the electrode. Withdrawal of the electrode broke the tube not the soldering bond (a). Next the tube ends were soldered together (b) and again withdrawal of the electrode resulted in a broken tube. $180^{\circ} \mathrm{C}$, which further reduced the resistivity by $2-3$ orders of magnitude. The results reported in this paper were obtained at room temperature without annealing.

We observed a decrease in resistivity of the solder material bridges over time when passing current through the bridge. The decrease went faster when increasing the current. This could be evidence of an annealing process similar to the observations described above. This is subject to ongoing investigations.

The method of nanosoldering presented here does not depend on the particular nanocomponent nor on the electrode material. It involves no lithographic steps and demands no electrical connections such as in spot-welding [5], and it is straightforward to achieve accurate alignment. The nanosoldering technique could be central in assembly of 3D nanostructures. At present we are developing electrostatically actuated microfabricated tweezers equipped with nanoscale electron beam deposited tips [18]. The combination of such tweezers and the nanosoldering technique could be a route to integration of nanoscale components in microfabricated devices.

\section{ACKNOWLEDGMENT}

We acknowledge financial support from STVF (NANOHAND talent project).

\section{REFERENCES}

[1] P.G. Collins, P. Avouris, Sci. Am. Vol. 283, p. 38, 2000.

[2] P.A. Williams, S.J. Papadakis, M.R. Falvo, A.M. Patel, M. Sinclair, A. Seeger, A. Helser, R.M. Taylor, S. Washburn, R. Superfine, Appl. Phys. Lett., Vol. 80, p. 2574, 2002.

[3] M.F. Yu, M.J. Dyer, G.D. Skidmore, H.W. Rohrs, X.K. Lu, K.D. Ausman, J.R. Von Ehr, R.S. Ruoff, Nanotechnology, Vol. 10, p. $244,1999$.

[4] F. Banhart, Nano Lett., Vol. 1, p. 329, 2001.

[5] J. Cumings, A. Zettl, Science, Vol. 289, p. 602, 2000.

[6] A. Star, J.-C.P. Gabriel, K. Bradley, G. Gruner, Nano Lett., Vol. 3, p. 47, 2003.

[7] S.J. Tans, A.R.M. Verschueren, C. Dekker, Nature, Vol. 393, p. 49, 1998.

[8] H.W.P. Koops, C. Schossler, A. Kaya, M. Weber, J. Vac. Sci. Tech. B, Vol. 14, p. 4105, 1996.

[9] X.B. Wang, Y.Q. Liu, D.B. Zhu, Chem. Comm., p. 751, 2001.

[10] P. Bøggild, T.M. Hansen, C. Tanasa, F. Grey, Nanotechnology, Vol. 12, p. $331,2001$.

[11] D.N. Madsen, K. Mølhave, R. Mateiu, A.M. Rasmussen, M. Brorson, C.H.J. Jacobsen, P. Bøggild, Nano Lett., Vol. 3, p. 47, 2003.

[12] K. Melhave, D.N. Madsen, A.M. Rasmussen, A. Carlsson, C.C. Appel, M. Brorson, C.H.J. Jacobsen, P. Bøggild, "Solid gold nanostructures fabricated by electron beam deposition", Submitted to Nano Lett.

[13] F. Banhart, Rep. Prog. Phys., Vol. 62, p. $1181,1999$.

[14] A.V. Krasheninnikov, K. Nordlund, J. Keinonen, Phys. Rev. B, Vol. 65, $165423,2002$.

[15] S. Franks, P. Poncharal, Z.L. Wang, W.A. Heer, Science, Vol. 280, p. $1744,1998$.

[16] P.G. Collins, P. Avouris, Appl. Phys. A, Vol. 329, p.74, 2002.

[17] A. Bietsch, B. Michel, Appl. Phys. Lett., Vol. 80, p. 3346, 2002.

[18] K. Mølhave, T.M. Hansen, D.N. Madsen and P. Bøggild, "Towards pick-and-place assembly of nanostructures", J. Nanosci. Nanotech., in press. 